\title{
EDA Çekirdekli Amin, TRIS ve Karboksil Sonlu PAMAM Dendrimerleri Kullanarak Ketoprofenin Çözünürlüğünü Geliștirme
}

\author{
Ali Serol ERTÜRK'1, Mustafa Ulvi GÜRBÜZ ${ }^{* 2}$ \\ ${ }^{1}$ Adıyaman Üniversitesi, Eczacılık Fakültesi, Eczacılık Temel Bilimler Bölümü, 02040, Adıyaman \\ ${ }^{2}$ Yıldız Teknik Üniversitesi, Fen Edebiyat Fakültesi, Kimya Bölümü, 34349, İstanbul
}

(Alınış / Received: 19.05.2017, Kabul / Accepted: 22.09.2017, Online Yayınlanma / Published Online: 24.10.2017)

Anahtar Kelimeler

Dendrimer,

Poli(amidoamine) PAMAM,

İlaç taşıyıcl,

Ketoprofen
Özet: Steroid olmayan antienflamatuar (NSAİ) ilaçlar ağrı kesici, ateş düşürücü ve antiinflamatuar etkilerinden dolayı yaygın bir şekilde kullanılmaktadırlar. Son yıllarda NSAİ ilaçların iyi bilinen klasik etkilerinin yanında birçok farklı terapötik etkilerinin (kanser, Alzheimer ve Parkinson hastalıkları) de bulunduğu keşfedilmiştir. Sonuçlar gösteriyor ki, poli(amidoamin) (PAMAM) dendrimerler varlığında ketoprofen'in (KETO) sudaki çözünürlüğü jenerasyon büyüklüğü (E2E4) ve dendrimer konsantrasyonun $(0-2 \mathrm{mM})$ artmasıyla önemli ölçüde geliştirilmiştir. KETO'nun $(0.22 \pm 0.003 \mathrm{mg} / \mathrm{mL})$ çözünürlüğünü arttırmada PAMAM dendrimerlerin rolü E4.TRIS $(52.77 \pm 2.06 \mathrm{mg} / \mathrm{mL})>$ E4.COOH $(36.42 \pm$ $0.54 \mathrm{mg} / \mathrm{mL})>$ E3.TRIS $(13.70 \pm 0.17 \mathrm{mg} / \mathrm{mL})>$ E3.COOH $(11.97 \pm 0.14 \mathrm{mg} / \mathrm{mL})>$ E4. $\mathrm{NH}_{2}(6.53 \pm 0.19 \mathrm{mg} / \mathrm{mL})>$ E2.COOH $(5.95 \pm 0.10 \mathrm{mg} / \mathrm{mL})>$ E2.TRIS $(5.72 \pm 0.10$ $\mathrm{mg} / \mathrm{mL})>\mathrm{E}^{2} . \mathrm{NH}_{2}(4.21 \pm 0.04 \mathrm{mg} / \mathrm{mL})>\mathrm{E} 2 . \mathrm{NH}_{2}(2.35 \pm 0.04 \mathrm{mg} / \mathrm{mL})$ sırasına göre ve $0.002 \mathrm{M}$ dendrimer varlığında 11 ile 240 kat aralığındadır.

\section{Solubility Enhancement of Ketoprofen Using EDA Core Amine, TRIS and Carboxyl- Terminated PAMAM Dendrimers}

Keywords

Dendrimer,

Poly(amidoamin) PAMAM,

Drug carrier,

Ketoprofen

\begin{abstract}
Non-steroidal anti-inflammatory drugs (NSAIDs) are widely used for their analgesic, antipyretic and anti-inflammatory functions. Besides their well known classical functions, in recent years, many other novel therapeutic actions (cancer, Alzheimer and Parkinson diseases) of the NSAIDs have been discovered. Results showed that the aqueous solubility of ketoprofen (KETO) in the presence of poly(amidoamine) (PAMAM) dendrimers improved significantly with an increasing generation size (E2-E4) and dendrimer concentration $(0-2 \mathrm{mM})$. The role of PAMAM dendrimers in the solubility enhancement of KETO $(0.22 \pm 0.003$ $\mathrm{mg} / \mathrm{mL})$ was in the order of E4.TRIS $(52.77 \pm 2.06 \mathrm{mg} / \mathrm{mL})>$ E4.COOH $(36.42 \pm$ $0.54 \mathrm{mg} / \mathrm{mL})>$ E3.TRIS $(13.70 \pm 0.17 \mathrm{mg} / \mathrm{mL})>\mathrm{E} 3 . \mathrm{COOH}(11.97 \pm 0.14 \mathrm{mg} / \mathrm{mL})>$ E4.NH $2(6.53 \pm 0.19 \mathrm{mg} / \mathrm{mL})>$ E2.COOH $(5.95 \pm 0.10 \mathrm{mg} / \mathrm{mL})>$ E2.TRIS $(5.72 \pm 0.10$ $\mathrm{mg} / \mathrm{mL})>\mathrm{E} 3 . \mathrm{NH}_{2}(4.21 \pm 0.04 \mathrm{mg} / \mathrm{mL})>\mathrm{E} 2 . \mathrm{NH}_{2}(2.35 \pm 0.04 \mathrm{mg} / \mathrm{mL})$, and in the ranges of 11 to 240 -fold in the presence of $0.002 \mathrm{M}$ dendrimer.
\end{abstract}

\section{Giriş}

Steroid olmayan antienflamatuar (NSAí) ilaçlar eklem ve kronik kas iskelet sistemlerindeki ağrıların tedavisinde sıklıkla kullanılmasının yanı sıra kolon kanserinin gelişimine karşı koruyucu bir etki göstermektedir $[1,2]$. NSAİ ilaçların yan etkilerinin (gastrointestinal ve renal fonksiyonel bozukluğu) ve çözünürlük problemlerinin olması sebebiyle klinik uygulamaları sınırlı kalmaktadır. Bu nedenle, NSAİ ilaçların çözünürlüğünü ve biyoyararlanımını artırmak için ilaçlara yüzey aktif ajanlar eklenmekte ya da suda çözünebilen tuzları haline getirilmektedir. $[3,4,5]$. Ancak ilaç üzerinde yapılan en ufak bir formülasyon değişikliği bile ilacın etkisinin azalmasına neden olmaktadır [6].

Düşük çözünürlük ve biyoyararlığa sahip ilaçların vücutta arzu edilen bir aralıkta salımını gerçekleştirmek, sağllklı dokulara olan etkileri minimize etmek için lipozom, nanopartiküller, miseller, dendrimer ve siklodekstrin gibi çeşitli ilaç taşıyıcı sistemler kullanılmaktadır $[7,8]$. İlaç taşıyıcı sistemlerin vücut içerisinde kararlı olmaması, sağlıklı 
dokularla etkileşime girmesi, nano boyutlarının böbrek emilimine izin verecek büyüklükte olması ve bu nedenle kan dolaşımında kalmayarak hızlı bir şekilde dışarı atılması gibi bir çok problemlerle karşı karşıya kalınmaktadır [9, 10]. Bu problemlerden kaçınmak için dendrimerler potansiyel bir ilaç taşıyıcı sistemler olarak ön plana çlkmaktadır [11]. Dendrimerlerin boyutlarının ve yüzey gruplarının kontrol edilebilir olması, molekül içi boşlukların fazla olması sebebiyle ilaç taşıyıcı sistemler içinde önemli bir yer edinmektedir $[12,13]$. Dendrimerinlerin sahip olduğu çekirdek, tekrar eden dallar, uçlarda bulunan fonksiyonel grupların yanı sıra jenerasyon, konsantrasyon ve $\mathrm{pH}$ ilaçların çözünürlüğünü arttırmada etkilidirler [14]. Dendrimerler, suda çok az çözünen birçok ilaçların (antikanser, antimalaryal, antiviral, antitüberküler, antimikrobiyal, NSAIII ve antihipertansif) çözünürlüklerini arttırmada ve kontrollü olarak salımını gerçekleştirmekle birlikte, ilacın vücutta etkinliğini azaltmadan istenilen yere tașıyabilmesi büyük avantajlarındandır [15]. Bu avantajları göstermesinde en önemli üç etkenin etkili olduğu ve bunlardan birinci etkenin, ilaç dendrimerin yüzeyine kovalent olarak bağlanabilir olması; ikinci etken, ilaç dendrimerin diş yada iç gruplarla iyonik olarak etkileşerek koordine olabilmesi; üçüncü etken ise dendrimer ilaç molekülünü tamamen içine alacak şekilde enkapsüle eder ve tek molekülmüş gibi davranır [16].

Son zamanlarda etilendiamin (EDA) çekirdekli amin sonlu dendrimerler: Niklozamit [17], Nifedipin [18], Ketoprofen [19], Doksorubisin [20], Kandesartan Sileksetil [21] gibi ilaçların çözünürlüğünü arttırmada kullanılmıștır. Dendrimerler farmasötik alanda geniş uygulama alanına sahip olmasına rağmen, amin sonlu (PAMAM-NH2) dendrimerler vücut içerisinde katyonik forma dönüşerek toksik özellik göstermesinden dolayı biyolojik sistemlerde kullanımı kısıtlanmaktadır. Dendrimerin yüzeyinde bulunan katyonik yüzey gruplarının artmasıyla, biyolojik membrandaki negatif yüklerle etkileşerek membran yüzeyin bozulmasına, incelmesine ve hücrenin ölmesine sebebiyet vermektedir [15, 22]. Fakat karboksil sonlu (PAMAM-COOH) dendrimerler ise katyonik dendrimere kıyasla daha az toksik ya da B16F10, CCRF ve HepG2 gibi hücrelere karşı toksik olmadığı tespit edilmiştir [23]. İlaç taşıyıcı sistemlerde kullanılmak üzere PAMAM-COOH dendrimerine alternatif olarak Twyman vd. Tris (hidroksimetil) aminometan (TRIS) sonlu PAMAM dendrimer sentezleyerek, küçük asidik hidrofobik ilaçların çözünürlüklerinin arttırdığını göstermiştir [24].

Son çalışmamızda EDA [21] çekirdekli farklı jenerasyon (G2-G4) ve yüzey fonksiyonel grubuna $\left(\mathrm{NH}_{2}, \mathrm{COOH}\right.$ ve TRIS $\left(\mathrm{C}\left(\mathrm{CH}_{2} \mathrm{OH}\right)_{3}\right)$ sahip olan PAMAM dendrimerlerin sentezini ve karakterizayonunu gösterdik. $\mathrm{Bu}$ çalışmada EDA çekirdekli yüksek jenerasyonlu amin $\left(\mathrm{NH}_{2}\right)$, asit $(\mathrm{COOH})$ ve TRIS sonlu suda çözünebilen PAMAM dendrimerlerin, sudaki çözünürlüğü zayıf ve bundan dolayı canlı hücrelerde biyoyararlanımları düşük olan ketoprofen (KETO) (Şekil 1) ile fiziksel etkileşimlerini sağlayarak ilacın sudaki çözünürlüğü araştırıldı.

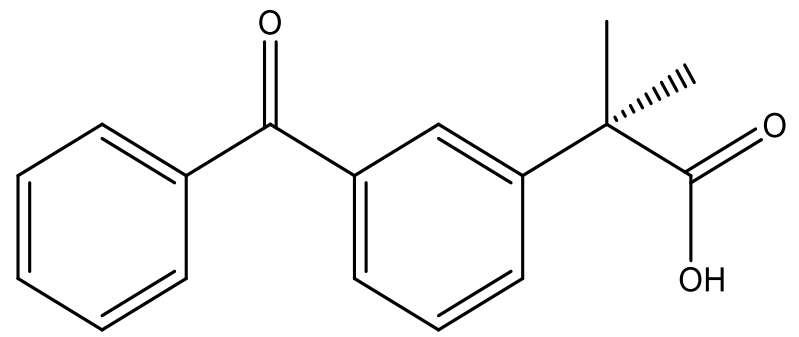

Şekil 1. KETO kimyasal özellikleri: $\lambda_{\max }=261 \mathrm{~nm}, \mathrm{pKa}=$ 4.45 , Moleküler ağırlık $=254.28 \mathrm{~g} / \mathrm{mol}$

\section{Materyal ve Metot}

\subsection{Materyaller ve cihazlar}

Tris (hidroksimetil) aminometan (TRIS), etilendiamin (EDA), metilakrilat, metanol, n-butanol, $\mathrm{NaOH}, \mathrm{K}_{2} \mathrm{CO}_{3}$ ve ketoprofen (KETO) Sigma-Aldrich firmasından temin edildi, Bütün çözeltiler 18.2 M $\Omega$ Millipore MilliQ cihazından elde edilen ultra saf ile hazırland. UVVis spektrumları PG T70 UV-Vis spektrofotometresi kullanılarak alındı. İnfrared spektrum ölçümleri için ATR-FTIR Bruker Tensor 27 (4000-400 $\left.\mathrm{cm}^{-1}\right)$ spektrofotometresi kullanıldı.

\subsection{PAMAM dendrimerin sentezi}

Mikrodalga destekli sentez yöntemi kullanılarak EDA çekirdekli PAMAM-NH 2 (E2-E4.NH2), PAMAM-TRIS (E2-E4.TRIS) ve PAMAM-COOH (E2-E4.COOH) dendrimerlerin sentezi son çalışmamızda gösterildi [21].

\section{3. İlaç-PAMAM dendrimer komplekslerinin hazırlanması ve karakterizasyonu}

\subsection{1. İlaç-PAMAM dendrimer komplekslerin hazırlanması}

KETO-PAMAM dendrimer kompleksleri önceki çalışmalarımıza göre hazırlandı $[21,25,26]$. 1:1 molar oranında KETO-PAMAM karışımları metanol'de çözüldü. 24 saat süre boyunca $37{ }^{\circ} \mathrm{C}$ sıcaklıkta ve $250 \mathrm{rpm}$ hızında karıştırıldı. Döner buharlaștırıcı yardımıyla çözücü uzaklaştırıldı ve geriye kalan çökeltiye saf su ilave ederek 2 saat boyunca karışması sağlandı. Daha sonra $0.45 \mu \mathrm{m}$ 'lik gözeneklere sahip süzgeç kâğıdı ile filtre edildi. KETO-PAMAM dendrimer kompleksleri ATR-FTIR (4000-600 $\left.\mathrm{cm}^{-1}\right)$ ve UV-Vis (400-200 nm) cihazları ile analizi yapıldı.

\subsubsection{UV-Vis karakterizasyonu}

KETO-PAMAM dendrimer kompleksleri metanol ile son konsantrasyonları $15 \mu \mathrm{g} / \mathrm{mL}$ olacak şekilde seyreltildi. Bu komplekslerin UV-Vis cihazı ile 400- 
$200 \mathrm{~nm}$ aralığında spektrum ölçümleri alındı. KETO karakteristik bantı $\lambda_{\max }=261 \mathrm{~nm}$ 'dir. KETO-PAMAM dendrimer kompleksleri oluștuktan sonra bu bantlarda herhangi bir kayma gözlemlenmedi [21, 25, 26].

\subsubsection{ATR-FTIR spektral çalışması}

PAMAM-TRIS dendrimer, KETO ve KETO-PAMAMTRIS dendrimer komplekslerinin ATR-FTIR (4000$600 \mathrm{~cm}^{-1}$ ) spektrumları $4 \mathrm{~cm}^{-1}$ de 20 tarama yapılarak çekildi.

\subsection{KETO çözünürlük testi}

Farklı jenerasyon ve yüzey gruplarına sahip PAMAM dendrimerlerin $2.5 \times 10^{-4}-2 \times 10^{-3} \mathrm{M}$ arasindaki ultra saf su içerisindeki çözeltileri $5 \mathrm{~mL}$ 'lik balon jojede hazırlandı. Fazla miktarda ilaç ilave edilerek doygun çözeltiler oluşturulduktan sonra 24 saat boyunca 37 oC'de 250 rpm hızında karıștırıldı. 24 saatin sonunda çözeltiler $6000 \mathrm{rpm}$ hızında 5 dakika boyunca santrifüj yapıldıktan sonra çözünmeyen ilaçlar 0.45 $\mu$ m'lik gözeneklere sahip süzgeç kağıdı ile çözelti ortamından uzaklaștırıldı. KETO-PAMAM dendrimer kompleks çözeltilerinin absorbans bantları belli bir aralıkta (0-1 abs) olabilmesi için seyreltme yapıldıktan sonra ölçümler alındı. KETO'nun kalibrasyon eğrisinden faydalanılarak ilacın miktarı belirlendi.

\section{Tartışma ve Sonuç}

\subsection{KETO-PAMAM dendrimer komplekslerinin UV-Vis ve ATR-FTIR tarafindan spektroskopik karakterizasyon}

\subsubsection{UV-Vis karakterizasyonu}

KETO, PAMAM dendrimer ve KETO-PAMAM dendrimer komplekslerinin UV-Vis spektrumları Şekil 2'de gösterilmektedir. KETO için $\lambda_{\max }=261 \mathrm{~nm}$ iken PAMAM dendrimerlerin $\lambda_{\max }=\sim 280 \mathrm{~nm}$ dir. KETO-PAMAM dendrimer komplekslerinin ve KETO'nun 261 nm'deki absorbans bantları gösteriyor ki, dendrimerler KETO ile elektrostatik etkileşim ve enkapsülasyon yaparak KETO'nun çözünürlüğünün artmasına neden olmuşlardır [27].

\subsubsection{ATR-FTIR karakterizasyonu}

KETO, E3.TRIS ve KETO-E3.TRIS dendrimer komplekslerinin ATR-FTIR spektrumları Şekil 3'te gösterilmektedir. KETO karakteristik bantları: 2977, $2937 \mathrm{~cm}^{-1}$ (C-H); 1697, $1652 \mathrm{~cm}^{-1}$, (C=0); 1597, 1576, $1440 \mathrm{~cm}^{-1} \quad(\mathrm{C}=0) \quad$ [28]. E3.TRIS dendrimerin karakteristik bantları ise: $\mathrm{O}-\mathrm{H}\left(3257 \mathrm{~cm}^{-1}\right)$, C-O $(1026$ $\left.\mathrm{cm}^{-1}\right), \mathrm{C}=\mathrm{O}\left(1643 \mathrm{~cm}^{-1}\right)$ ve N-H $\left(1560 \mathrm{~cm}^{-1}\right), \mathrm{C}-\mathrm{O}(1392$ $\mathrm{cm}^{-1}$ ). PAMAM dendrimerin KETO ile kompleks oluşturduktan sonra karakteristik bantlarında, PAMAM dendrimerin polar grupları ile hidrofilik ve elekrostatik etkileşimi sonucunda $0-15 \mathrm{~cm}^{-1}$ spektral kaymalar gözlemlendi. Bu spektral kaymayalar, ilaç molekülünün $\mathrm{COOH}$ grubu ile dendrimerin iç kavitelerinde ve yüzeyinde bulunan amit ve hidroksil grupların hidrofilik etkileșimlerinin katkıda bulunduğu söylenebilir [29, 30].

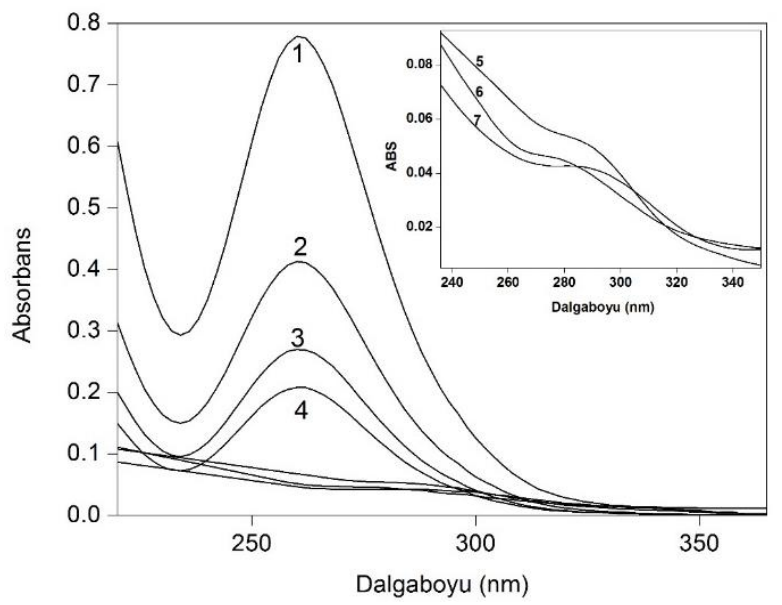

Şekil 2. UV-Vis spektrumu KETO $(15 \mu \mathrm{g} / \mathrm{mL})$; (2) KETO-E4. TRIS kompleks (15 $\mu \mathrm{g} / \mathrm{mL})$; (3) KETO-E4.COOH kompleks (15 $\mu \mathrm{g} / \mathrm{mL})$; (4) KETO-E4.NH 2 kompleks (15 $\mu \mathrm{g} / \mathrm{mL})$; (5) $\mathrm{E} 4 . \mathrm{COOH}(50 \mu \mathrm{g} / \mathrm{mL}) ;(6) \mathrm{E}^{2} \mathrm{NH}_{2}(50 \mu \mathrm{g} / \mathrm{mL}) ;(7)$ E4.TRIS $(50 \mu \mathrm{g} / \mathrm{mL})$

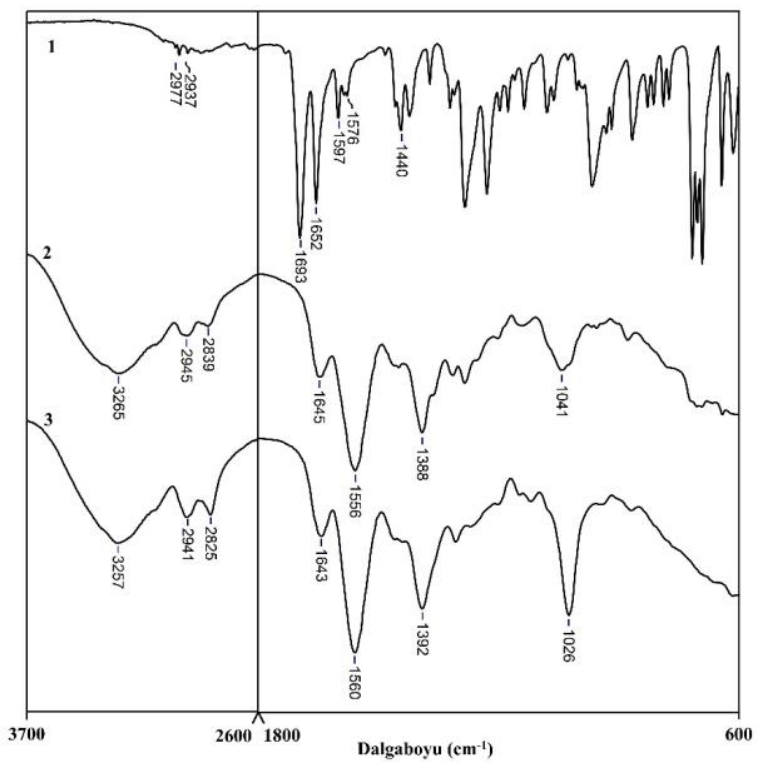

Sekil 3. EDA çekirdekli PAMAM dendrimerin KETO ile yapmış olduğu komplekslerin ATR-FTIR spektrumları (1) KETO; (2) E3.TRIS-KETO; (3) E3.TRIS

\subsection{KETO çözünürlüğü üzerindeki dendrimerin konsantrasyon ve jenerasyon etkisi}

Farklı konsantrasyon ve jenerasyona sahip EDA çekirdekli TRIS, $\mathrm{COOH}$ ve $\mathrm{NH}_{2}$ sonlu suda çözenebilen PAMAM dendrimerlerin KETO'nun sudaki çözünürlük çalışmaları yapılmış ve sonuçlar Şekil 4'de gösterilmiştir. KETO'nun sudaki çözünürlüğünde, dendrimerlerin jenerasyon (G2-G4) ve konsantrasyonların (0-2 mM) artmasıyla önemli bir artış tespit edilmiştir. Bu sonuçlar bize gösteriyor ki, dendrimerlerin jenerasyon sayısı arttıkça iç kavitelerinin ve ilacın elektrostatik etkileşimde 
bulunabileceği fonksiyonel gruplarında (primer ve tersiyer amin gibi) sistematik bir șekilde artmasıyla KETO’nun çözünürlüğününde önemli bir artış sağlandı̆̆ gözlemlenmiştir.
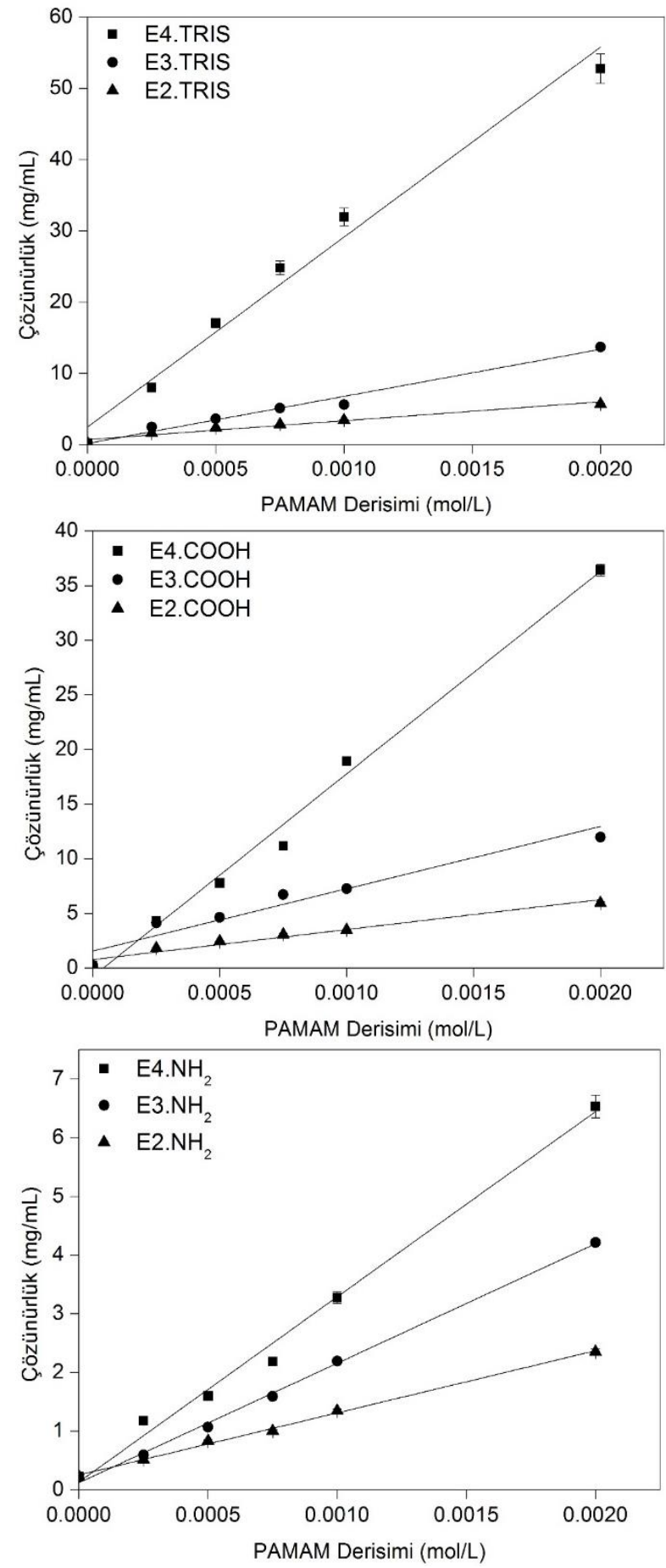

Şekil 4. $\mathrm{COOH}$, TRIS ve $\mathrm{NH}_{2}$ sonlu PAMAM dendrimerlerin jenerasyon ve konsantrasyona bağlı olarak KETO'nun çözünürlüğü üzerindeki etkisi

\subsection{KETO çözünürlüğü üzerindeki yüzey fonksiyonel grupların etkisi}

Dendrimerlerin büyüklüklerinin ve yüzey fonksiyonel gruplarının kontrol edilebilir olmasından dolayı ilaç taşıyıcı sistemlerde sıklıkla kullanılmaktadır. Amin sonlu dendrimerler suda çözünebilir olması bir avantaj gibi gözükse de, vücut içerisinde pozitif yüklü olmasından dolayı negatif yüklü hücrelerle etkileşerek hücrenin ölmesine sebebiyet vermektedir. İlaç çalışmalarında daha az toksik olan karboksil sonlu negatif yüklü dendrimerler kullanılmasının yanı sıra nötr yüklü TRIS sonlu dendrimerlerin daha az sitotoksisite gösterebileceği bilinmektedir [15]. PAMAM dendrimerin yüzeyinde ve iç kavitelerinde bulunan birçok fonksiyonel gruplar, ilaçlarla elektrostatik etkileşim yaparak etken maddenin çözünürlüğünün artmasına sebep olmaktadır. KETO'nun sudaki çözünürlüğünü ve canlı hücrelerde biyoyararlanımlarını arttırmak için miseller (0.1 mg/mL'den $19.60 \mathrm{mg} / \mathrm{mL}^{\prime} \mathrm{e}$ ) [31], poli propil eter imin (PETIM) [32] (sudaki çözünürlüğüne göre 5 kat arttırmış) ve EDA çekirdekli amin sonlu PAMAM dendrimerler [19] (sudaki çözünürlüğüne göre 2-4 kat arttırmış) kullanılmıştır. Farklı yüzey gruplarına sahip dendrimerlerin KETO'nun çözünürlüğü üzerindeki etkisi Şekil 4'te gösterilmiştir. Elde ettiğimiz sonuçlara göre TRIS sonlu PAMAM dendrimerlerin KETO'nun $(0.22 \pm$ $0.003 \mathrm{mg} / \mathrm{mL}$ ) çözünürlüğünü arttırmada önemli derecede katkı sağladığı, amin sonlu PAMAM dendrimerlerin ise en az etki sağladığ gözlemlenmiştir (E4.TRIS $(52.77 \pm 2.06 \mathrm{mg} / \mathrm{mL})>\mathrm{E} 4$. $\mathrm{COOH}(36.42 \pm 0.54 \mathrm{mg} / \mathrm{mL})>\mathrm{E}_{4} . \mathrm{NH}_{2}(6.53 \pm 0.19$ $\mathrm{mg} / \mathrm{mL})$ ). KETO'nun küçük molekül boyutlarına sahip olması PAMAM dendrimerlerin iç kavitelerine enkapsülasyonunu kolaylaştırmakla birlikte, yapısında $\mathrm{COOH}$ grubun dendrimerlerle (amin, hidroksil ve karboksil gibi) elektrostatik etkileşim yaparak çözünürlüğünün artmasına neden olmuştur. $\mathrm{Bu}$ çalışmanın sonuçlar bize gösteriyor ki, sentezi zahmetli ve zor olan TRIS sonlu PAMAM dendrimerleri grubumuz tarafından hızlı ve yüksek safllkta sentezleyerek, TRIS ve asit sonlu PAMAM dendrimerlerin potansiyel bir ilaç taşıyıcı sistemler olacağı düşünülmektedir.

\section{Kaynakça}

[1] Griffin, M.R. 1998. Epidemiology of Nonsteroidal Anti-Inflammatory Drug-Associated Gastrointestinal Injury. The American Journal of Medicine, 104(1998), 23-29.

[2] Chan, T.A. 2002. Nonsteroidal AntiInflammatory Drugs, Apoptosis, and ColonCancer Chemoprevention. The Lancet Oncology, 3(2002), 166-174.

[3] Polisson, R. 1996. Nonsteroidal AntiInflammatory Drugs: Practical and Theoretical Considerations in Their Selection. The American Journal of Medicine, 100(1996), 31S-36S.

[4] Fujii, M., Hori, N., Shiozawa, K., Wakabayashi, K., Kawahara, E., Matsumoto, M. 2000. Effect of Fatty Acid Esters on Permeation of Ketoprofen Through Hairless Rat Skin. International Journal of Pharmaceutics, 205(2000), 117-125.

[5] Vergote, G.J., Vervaet, C., Van Driessche, I., Hoste, S., De Smedt, S., Demeester, J., Jain, R.A., Ruddy, 
S., Remon, J.P. 2001. An Oral Controlled Release Matrix Pellet Formulation Containing Nanocrystalline Ketoprofen. International Journal of Pharmaceutics, 219(2001), 81-87.

[6] Beezer, A., King, A., Martin, I., Mitchel, J., Twyman, L., Wain, C. 2003. Dendrimers as Potential Drug Carriers; Encapsulation of Acidic Hydrophobes Within Water Soluble PAMAM Derivatives. Tetrahedron, 59(2003), 3873-3880.

[7] Felice, B., Prabhakaran, M.P., Rodríguez, A.P., Ramakrishna, S. 2014. Drug Delivery Vehicles on A Nano-Engineering Perspective. Materials Science and Engineering: C, 41(2014), 178-195.

[8] Sahoo, S.K., Dilnawaz, F., Krishnakumar, S. 2008. Nanotechnology in Ocular Drug Delivery. Drug Discovery Today, 13(2008), 144-151.

[9] Vandamme, T.F., Brobeck, L. 2005. Poly (amidoamine) Dendrimers as Ophthalmic Vehicles for Ocular Delivery of Pilocarpine Nitrate and Tropicamide. Journal of Controlled Release, 102(2005), 23-38.

[10] Fox, M.E., Szoka, F.C., Fréchet, J.M.J. 2009. Soluble Polymer Carriers for the Treatment of Cancer: The Importance of Molecular Architecture. Accounts of Chemical Research, 42(2009), 11411151.

[11] Jia, L., Xu, J.P., Wang, H., Ji, J. 2011. Polyamidoamine Dendrimers SurfaceEngineered with Biomimetic Phosphorylcholine as Potential Drug Delivery Carriers. Colloids and Surfaces B-Biointerfaces, 84(2011), 49-54.

[12] Malik, N., Wiwattanapatapee, R., Klopsch, R., Lorenz, K., Frey, H., Weener, J.W., Meijer, E.W., Paulus, W., Duncan, R. 2000. Dendrimers: Relationship Between Structure and Biocompatibility in Vitro, and Preliminary Studies on the Biodistribution of 125I-Labelled Polyamidoamine Dendrimers in Vivo. Journal of Controlled Release, 65(2000), 133-148.

[13] Rai, A.K., Tiwari, R., Maurya, P., Yadav, P. 2016. Dendrimers: A Potential Carrier for Targeted Drug Delivery System. Pharmaceutical and Biological Evaluations, 3(2016), 275-287.

[14] Mignani, S., Kazzouli, S.A., Bousmina, M., Majoral, J.P. 2013. Expand Classical Drug Administration Ways by Emerging Routes Using Dendrimer Drug Delivery Systems: A Concise Overview. Advanced Drug Delivery Reviews, 65(2013), 1316-1330.

[15] Kesharwani, P., Jain, K., Jain, N.K. 2014. Dendrimer as Nanocarrier for Drug Delivery. Progress in Polymer Science, 39(2014), 268307.

[16] Devarakonda, B., Li, N., de Villiers, M.M. 2005. Effect of Polyamidoamine (PAMAM) Dendrimers on The in Vitro Release of Water-Insoluble
Nifedipine from Aqueous Gels. AAPS PharmSciTech, 6(2005), E504-E512.

[17] Devarakonda, B., Hill, R.A., Liebenberg, W., Brits, M., de Villiers, M.M. 2005. Comparison of The Aqueous Solubilization of Practically Insoluble Niclosamide by Polyamidoamine (PAMAM) Dendrimers and Cyclodextrins. International Journal of Pharmaceutics, 304(2005), 193-209.

[18] Devarakonda, B., Hill, R., Devilliers, M. 2004. The Effect of PAMAM Dendrimer Generation Size and Surface Functional Group on The Aqueous Solubility of Nifedipine. International Journal of Pharmaceutics, 284(2004), 133-140.

[19] Yiyun, C., Tongwen, X., Rongqiang, F. 2005. Polyamidoamine Dendrimers Used as Solubility Enhancers of Ketoprofen. European Journal of Medicinal Chemistry, 40(2005), 1390-1393.

[20] Ke, W., Zhao, Y., Huang, R., Jiang, C., Pei, Y. 2008. Enhanced Oral Bioavailability of Doxorubicin in A Dendrimer Drug Delivery System. Journal of Pharmaceutical Sciences, 97(2008), 2208-2216.

[21] Ertürk, A.S., Gürbüz, M.U., Tülü, M. 2017. The Effect of PAMAM Dendrimer Concentration, Generation Size and Surface Functional Group on The Aqueous Solubility of Candesartan Cilexetil. Pharmaceutical Development and Technology, 22(2017), 111-121.

[22] Mecke, A., Majoros, I.J., Patri, A.K., Baker, J.R., Banaszak Holl, M.M., Orr, B.G., 2005. Lipid Bilayer Disruption by Polycationic Polymers: The Roles of Size and Chemical Functional Group. Langmuir, 21(2005), 10348-10354.

[23] Duncan, R., Izzo, L. 2005. Dendrimer Biocompatibility and Toxicity. Advanced Drug Delivery Reviews, 57(2005), 2215-2237.

[24] Twyman, L.J., Beezer, A.E., Esfand, R., Hardy, M.J., Mitchell, J.C. 1999. The Synthesis of Water Soluble Dendrimers, and Their Application as Possible Drug Delivery Systems. Tetrahedron Letters, 40(1999), 1743-1746.

[25] Ertürk, A.S., Gürbüz, M.U., Tülü, M. 2017. Newgeneration Jeffamine ${ }^{\circledR}$ D230 Core Amine, TRIS and Carboxyl-Terminated PAMAM Dendrimers: Synthesis, Characterization and The Solubility Application for A Model NSAID Drug Ibuprofen. Marmara Pharmaceutical Journal, 21(2017), 385-399.

[26] Gürbüz, M.U., Ertürk, A.S., Tülü, M. 2016. Synthesis of Surface-Modified TREN-Cored PAMAM Dendrimers and Their Effects on The Solubility of Sulfamethoxazole (SMZ) as An Analog Antibiotic Drug. Pharmaceutical Development and Technology, 2016), 1-12.

[27] Chauhan, A.S., Jain, N.K., Diwan, P.V., Khopade, A.J. 2004. Solubility Enhancement of Indomethacin with Poly(amidoamine) 
Dendrimers and Targeting to Inflammatory Regions of Arthritic Rats. Journal Drug Targeting, 12(2004), 575-583.

[28] Silion, M., Hritcu, D., Popa, M. 2010. Preparation and Characterization of Ketoprofen-Layered Double Hydroxide Compounds. Journal of Optoelectronics and Advanced Materials, 12(2010), 2151-2157.

[29] Popescu, M.C., Filip, D., Vasile, C., Cruz, C., Rueff, J., Marcos, M., Serrano, J., Singurel, G. 2006. Characterization by Fourier Transform Infrared Spectroscopy (FT-IR) and 2D IR Correlation Spectroscopy of PAMAM Dendrimer. The Journal of Physical Chemistry B, 110(2006), 1419814211.

[30] Singh, P., Gupta, U., Asthana, A., Jain, N.K. 2008. Folate and folate- PEG- PAMAM Dendrimers: Synthesis, Characterization, and Targeted Anticancer Drug Delivery Potential in Tumor Bearing Mice. Bioconjugate Chemistry, 19(2008), 2239-2252.

[31] Latere Dwan'Isa, J.P., Rouxhet, L., Preat, V., Brewster, M., Arien, A. 2007. Prediction of Drug Solubility in Amphiphilic Di-Block Copolymer Micelles: The Role of Polymer-Drug Compatibility. Die Pharmazie-An International Journal of Pharmaceutical Sciences, 62(2007), 499-504.

[32] Jain, S., Kaur, A., Puri, R., Utreja, P., Jain, A., Bhide, M., Ratnam, R., Singh, V., Patil, A., Jayaraman, N. 2010. Poly Propyl Ether Imine (PETIM) Dendrimer: A Novel Non-Toxic Dendrimer for Sustained Drug Delivery. European Journal of Medicinal Chemistry, 45(2010), 4997-5005. 